\title{
Pleomorphic adenoma of the buccal mucosa: a rare entity
}

\begin{abstract}
Pleomorphic adenoma is the most common salivary gland tumor, accounting for about $60 \%$ of all the salivary gland neoplasms. It mainly arises from the parotid glands and it involves the minor salivary glands in approximately $8 \%$ of the cases, with the hard palate being the most common site of its occurrence. We herein report a very rare case of pleomorphic adenoma of the buccal mucosa in a 40 -year-old female which posed as a diagnostic conundrum.
\end{abstract}

Keywords: buccal mucosa, minor salivary glands, pleomorphic adenoma
Volume 10 Issue $6-2019$

\author{
Sonam Sharma, 'Amita Sharma ${ }^{2}$ \\ 'Department of Pathology, Kalpana Chawla Government \\ Medical College, Karnal, Haryana India \\ 2Department of Dentistry, SHKM Government Medical College, \\ Mewat, Haryana India
}

Correspondence: Sonam Sharma, Department of Pathology, Kalpana Chawla Government Medical College, Karnal, Haryana, India,Tel 9999841393,Email drsonamsharma@gmail.com

Received: October 31, 2019 | Published: November 19, 2019
Abbreviations: USG, ultrasonography; CT, computed tomography; FNAC, fine needle aspiration cytology; MGG, maygrunwald-giemsa

\section{Introduction}

Salivary gland neoplasms account for less than $5 \%$ of all the tumors and represent $3-5 \%$ of all the head and neck tumors. ${ }^{1}$ The minor salivary gland tumors constitute for $22 \%$ of all salivary gland neoplasms with majority of them being malignant and only $18 \%$ benign. ${ }^{2,3}$ Pleomorphic adenoma is a benign mixed tumor which is the most common salivary gland tumor, accounting for $40-70 \%$ of all the tumors arising from major and minor salivary glands. ${ }^{4}$ Parotid gland is the most commonly affected major salivary gland. There are 800 to 1,000 minor salivary glands located throughout the oral cavity in the tissue of the buccal, labial and lingual mucosa, the soft palate, the hard palate, and the floor of the mouth. Among the minor salivary glands, palate is the commonly involved site of pleomorphic adenoma while the buccal mucosa or cheek is a rarely involved location. An extensive research of the pertinent world literature has revealed only a handful of documented cases related to pleomorphic adenoma arising from the buccal mucosa minor salivary glands. ${ }^{5}$ We describe one such rare case of pleomorphic adenoma of the buccal mucosa in a 40-year-old female which created a diagnostic dilemma. The approach to such a case, differential diagnosis and the management of this rare entity is also presented.

\section{Case report}

A 40-year-old female presented to the dental outpatient department with the chief complaint of mild swelling over the right side of her cheek since last 2 years. Her history of present illness revealed that initially a pea sized swelling was noticed by her inside the mouth on the right side of the cheek. It gradually grew over the last 6 months to attain the present size and was now associated with recurrent cheek biting. There was no history of bleeding, pain, sensory changes, and disturbance of salivation, fever, loss of appetite or weight loss. The past dental, medical and personal history was unremarkable. The family history was non-contributory. On general physical examination she was of moderate built and all her vitals were within the normal limits. There was no regional lymphadenopathy. No abnormality was detected on her systemic examination. On extraoral examination, there was slight facial asymmetry due to an oval swelling on the right side of the cheek. The overlying skin was normal. Opening of the mouth was adequate. Intraoral examination revealed the oral hygiene to be poor. A round to oval mass measuring $1.5 \times 1.5 \mathrm{~cm}$ in size was seen in the right buccal mucosa (Figure 1). On palpation, it was welldefined, firm in consistency, non-tender, non-fluctuant, non-reducible, non-pulsatile and freely movable. The color of the overlying mucosa was same as that of the adjacent mucosa. Local temperature over the swelling was not raised. On the basis of history and clinical diagnosis a provisional differential diagnosis of mucocoele or neurofibroma was made. Ultrasonography (USG) of the lesion was carried out, which showed a well-defined, soft tissue lesion in the right buccal region with solid and cystic components. Computed tomography (CT) scan of face was suggestive of a well-defined encapsulated homogeneously enhancing lesion in the right buccal space region without invasion of the adjacent structures. Both the radiological investigations suggested the possibility of a benign salivary gland neoplasm of the right buccal mucosa. Simultaneously, after her routine laboratory investigations to arrive at a conclusive diagnosis, Fine Needle Aspiration Cytology (FNAC) was performed. Two passes at two different sites of the lesion were done. Thick mucoid material was aspirated in both the attempts. This material was pushed onto the clean glass slides and the smears were drawn. The smears prepared, were stained with May-GrunwaldGiemsa (MGG) stain followed by microscopic examination. FNAC smears were cellular on microscopy and revealed distinct epithelial and mesenchymal components. The epithelial cells were round and had moderate amount of cytoplasm with a central round to oval bland nucleus having finely dispersed chromatin. The myoepithelial cells were larger and had moderate to abundant amount of pale blue cytoplasm and an eccentric round nucleus giving the cell a plasmacytoid appearance. Both these cell types were embedded within the chondromyxoid fibrillary stroma. There was no evidence of any nuclear atypia, prominent nucleoli or tumor diathesis (Figure 2). Based on these cytomorphological findings, a diagnosis of pleomorphic adenoma arising from the minor salivary glands of the right buccal mucosa was rendered. Excision of the lesion with adequate margin of the surrounding normal tissue was done under local anaesthesia and 
the specimen was sent for histopathological examination. Grossly, the mass was ovoid in shape, well-demarcated, partially encapsulated, grey-white, firm in consistency and measured around $1.7 \times 1.5 \times 0.5 \mathrm{~cm}$ in size. On cut section, it was greyish white with some gelatinous areas and foci of calcification. Formalin fixed paraffin embedded routine Hematoxylin and Eosin stained microsections revealed a well-circumscribed tumor comprising of proliferating epithelial cells, interspersed in chondromyxoid and fibrocollagenous stroma. Numerous nests of polygonal cells and interconnecting tubuloalveolar structures lined by two layers of cuboidal epithelial cells were seen. Ductal structures lined by a single layer of epithelial cells were also apparent. Cellular pleomorphism was absent (Figure 3). These histopathological findings pointed towards the final diagnosis of a benign pleomorphic adenoma of the minor salivary glands of the right buccal mucosa. The post-operative period of the patient was uneventful. There was no evidence of recurrence of the lesion during the 3 year follow-up period of the patient.

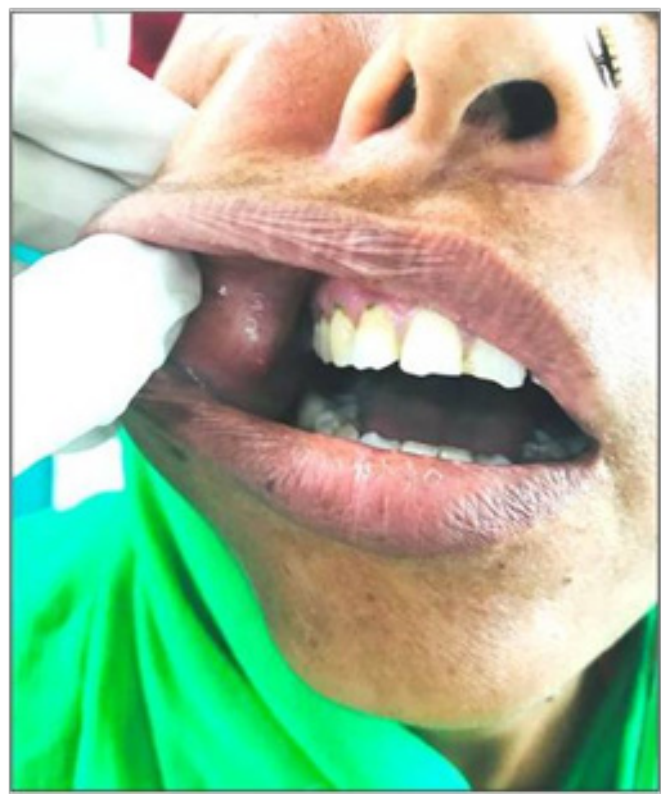

Figure I Intraoral submucosal nodule on clinical inspection.

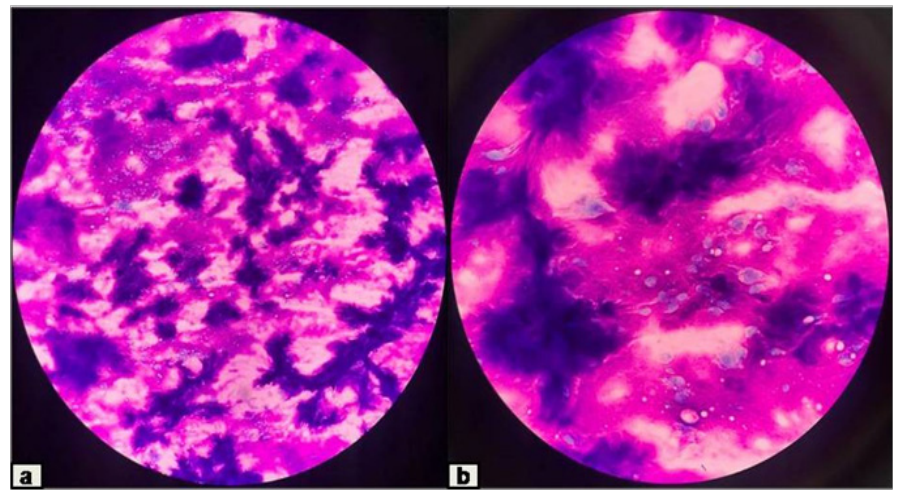

Figure 2 (A) Smear showing singly scattered and few groups of epithelial cells with interspersed chondromyxoid stromal fragments and background population of myoepithelial cells (MGG, $\times 40$ ).

(B) Metachromatic fibrillary stroma with associated embedded spindled myoepithelial cells (MGG, $x \mid 00)$.

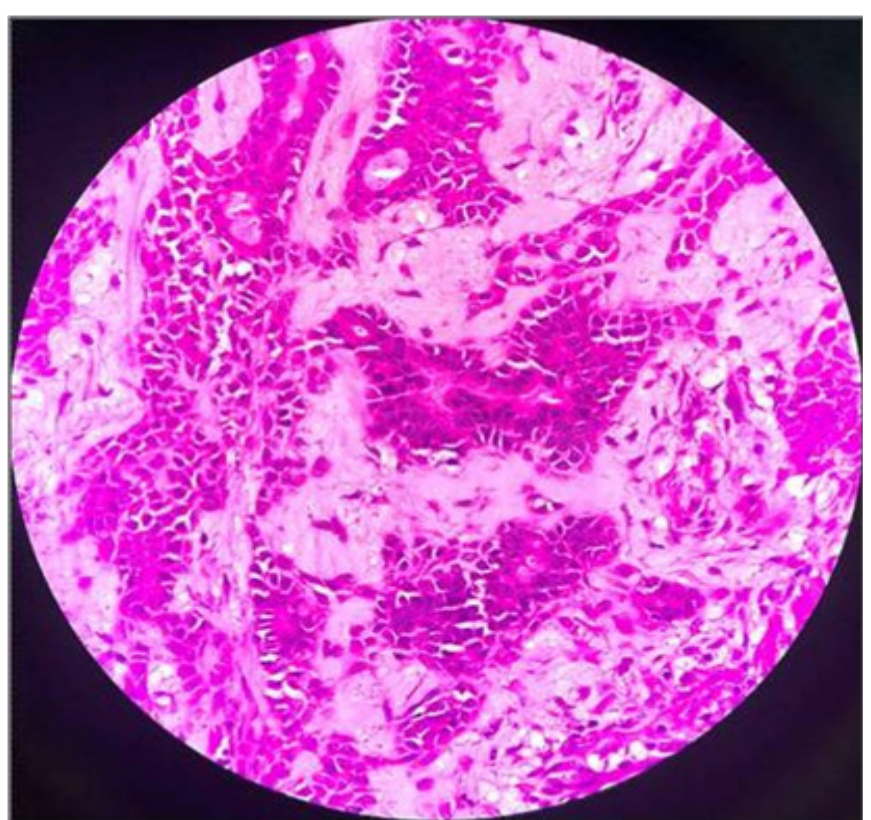

Figure 3 Photomicrograph exhibiting the epithelial cells with myxoid connective tissue stroma $(\mathrm{H} \& \mathrm{E}, \times 200)$.

\section{Discussion}

Salivary gland tumors are one of the most morphologically and clinically diverse group of neoplasms and about $80 \%$ of these are benign and innocuous. ${ }^{2,6}$ The etiopathogenesis of these tumors is still not well-defined, but few factors like radiation therapy, smoking, Epstein-Barr virus infection, genetic predisposition, sunlight, vitamin A deficiency and chemotherapy have been implicated in their occurrence. ${ }^{7}$ Amongst all the salivary gland tumors, pleomorphic adenoma is the most common salivary gland tumor that affects both the major and minor salivary glands. It arises mainly in the major salivary glands, mostly parotid (85\%), submandibular gland (8\%), and intraoral (7\%) salivary glands. Palatal glands are the most commonly involved minor salivary glands which are affected with pleomophic adenoma followed by the upper lip and cheek/buccal mucosa. Other rare sites include the throat, retromolar region, floor of the mouth, tongue, alveolar mucosa and nasal cavity. ${ }^{5,8}$

On further exploring the existing literature on pleomorphic adenoma arising from the buccal mucosa, certain clinicopathological features of this condition draw special attention, which in turn can help in its correct diagnosis and treatment. The pleomorphic adenoma of the buccal mucosa is known to arise from the submucosal buccal minor salivary glands. However, they are even known to arise from the glands located in the buccal space external to buccinator known as molar or buccal glands. ${ }^{9}$ Regarding its pathogenesis it is now generally accepted that pleomorphic adenoma is of epithelial origin and has been associated with clonal chromosome abnormalities with genetic aberrations involving $8 \mathrm{q} 12$ and $12 \mathrm{q} 15 .{ }^{10}$ Clinically, all the age groups can be involved. However, it most commonly occurs between the third to fifth decades of life and has a female predilection. The major presenting symptom is a painless, slow-growing, solitary, well-circumscribed, round to oval, firm, lobulated submucosal mass, the size of which can be variable and may range from 2 to $7 \mathrm{~cm}$ in greatest diameter, however, some tumors can attain massive sizes. 
The covering mucosa is seldom affected unless it is secondarily traumatized. ${ }^{11}$ Local discomfort is frequently present nevertheless, facial nerve involvement manifested by facial paralysis is rare. ${ }^{12}$ Once pleomorphic adenoma of buccal mucosa is clinically suspected, radiological investigations like USG, CT scan, Magnetic Resonance Imaging can be used for determining the size as well as the extent of the lesion and in assessing the bone involvement. Many researchers have documented that FNAC has been a boon for an early diagnosis of head and neck lesions including salivary gland neoplasm as it is not only a rapid, cost-effective diagnostic tool but it is also an excellent non-invasive method because in the presence of characteristic cytomorphology, it obviates the need for more invasive procedures. Ancillary tests like immunocytochemistry, cell block formation, liquid based cytology followed by histopathological examination and immunohistochemistry have also broadened the utilization of FNAC for the definite diagnosis of many tumors including those arising from salivary glands. ${ }^{13-23}$ Nevertheless, the histopathological examination of tissue obtained by surgical excision of the whole tumor is essential for the final diagnosis of pleomorphic adenoma of the buccal mucosa. The gross specimen is usually an encapsulated, solitary, well-defined, ovoid or round mass. Larger tumors may have a characteristic bosselated surface with necrotic or cystic regions. Its consistency varies from hard to rubbery to soft swelling that may be fluctuant. The cut surface of the tumor is solid and the color varies from gray blue, pale yellow to tan. Gritty areas and gelatinous or glistening foci can be evident. ${ }^{12}$ Histologically, pleomorphic adenoma is a combination of glandular epithelium and mesenchyme-like tissue and the proportion of each component varies widely among individual tumor. Researchers have categorized the tumor into the following types: principally myxoid, myxoid and cellular components present in equal proportions, predominantly cellular and extremely cellular. The epithelial components form ducts and small cysts that may contain an eosinophilic coagulum. The epithelium may also occur as small cellular nests, sheets of cells, anatomizing cords and foci of keratinizing squamous or spindle cells. Myoepithelial cells are responsible for giving the tumor a myxoid appearance and these cells can have variable morphology, sometimes appearing as angular or spindled, rounded with eccentric nuclei and hyalinized eosinophilic cytoplasm resembling plasma cells. The vacuolar degeneration of the myoepithelial cells can result in a cartilaginous appearance. Foci of hyalinization, bone and even fat can be noted in the connective tissue stroma of many tumors. ${ }^{24}$ In the minor glands, pleomorphic adenomas are often more solid or cellular than those seen in the major glands, and the myoepithelial cells are often polygonal with a pale eosinophilic cytoplasm giving an epithelioid or plasmacytoid phenotype..$^{25}$ The differential diagnosis of pleomorphic adenoma of the buccal mucosa includes buccal space abscess, hemangioma, dermoid cyst, lipoma, neurofibroma, rhabdomyosarcoma, mucoepidermoid carcinoma, adenoid cystic carcinoma, monomorphic adenoma, carcinoma ex pleomorphic adenoma, and foreign body reaction. ${ }^{26}$

Therapeutically, the pleomorphic adenoma of the minor salivary glands is best treated by wide surgical excision including a margin of normal tissue as it usually has a capsule that is either thin or incomplete, the extent of which will vary with tumor location, size, and histology., ${ }^{49}$ However, the intraoral lesions can be treated somewhat more conservatively by extracapsular excision. Inadequate resection, rupture of the tumor capsule, or tumor spillage following excision can lead to local recurrence as this tumor often has microscopic pseudopod-like extensions into the surrounding tissue due to dehiscence in the false capsule. Therefore, incisional biopsy is also avoided in such a rare scenario. ${ }^{27,28}$ Radiotherapy is not indicated due to the radio resistant nature of this tumor. A recurrence rate of 2 to $44 \%$ has been reported in the literature. ${ }^{12}$ In rare instances, about 1.6 to $7.5 \%$ of pleomorphic adenoma can undergo malignant transformation in its natural course and can give rise to carcinoma ex pleomorphic adenoma. ${ }^{29}$ Periodic long term follow-up pattern is mandatory as the rate of recurrence and malignant transformation even after many years of surgical excision is quite high. ${ }^{25}$

The current case was prompted to seek a medical treatment as she had a painless cheek mass which led to a noticeable cosmetic deformity and recurrent cheek bite. However, it was clinically mistaken as a mucocoele or neurofibroma. The radiological investigations though aided in the diagnosis but it was only on FNAC that the definite diagnosis of pleomorphic adenoma arising from the minor salivary glands of the right buccal mucosa could be made. Wide local excision was done for the tumor and the histopathological evaluation of resected specimen was consistent with the cytological diagnosis of pleomorphic adenoma of the right buccal mucosa. No recurrences or any fresh complaints have been reported so far by the patient even after 3 year of follow-up.

\section{Conclusion}

Pleomorphic adenoma of the buccal mucosa is a rare neoplasm which should always be kept in mind while dealing with cheek swellings. A high index of suspicion and radiological as well as cytological investigations play a pivotal role in clinching early definite diagnosis. However, the histopathological examination of the resected tumor obtained after complete wide surgical excision is essential. Tumor recurrence as well as malignant changes within the tumor itself is a concern which makes a long-term follow-up period necessary in all such cases.

\section{Acknowledgements}

None.

\section{Conflicts of interest}

No financial interest or any conflict of interest exists.

\section{References}

1. Eveson JW, Cawson RA. Tumours of the minor (oropharyngeal) salivary glands: A demographic study of 336 cases. J Oral Pathol. 1985;14(6):500-509.

2. Spiro RH. Salivary neoplasms: overview of a 35-year experience with 2,807 patients. Head Neck Surg. 1986;8(3):177-184.

3. Waldron CA, el-Mofty SK, Gnepp DR. Tumors of the intraoral minor salivary glands: a demographic and histologic study of 426 cases. Oral Surg Oral Med Oral Pathol. 1988; 66(3):323-333.

4. Jorge J, Pires FR, Alves FA, et al. Juvenile intraoral pleomorphic adenoma: report of five cases and review of the literature. Int $J$ Oral Maxillofac Surg. 2002;31(3):273-275.

5. Periasamy S, Manoharan A, Garg H, et al. Pleomorphic adenoma of the cheek: a case report. Cureus. 2019;11(8):e5312.

6. Sharma S, Chintamani, Khambra P, et al. Synchronous oral and breast malignancies: a rare presentation. Indian J Surg Oncol. 2017;8(3):426429 . 
7. Kulkarni HS, Kulkarni GH, Khaji SI, et al. Monomorphic adenoma: A rare benign neoplasm of the minor salivary gland: Case report and review of literature. J Dent Allied Sci. 2014;3(2):108-110.

8. Fidvi MZ, Jadhav AA, Kasatwar AP, et al. Pleomorphic adenoma of the cheek: Report of a rarity. J Datta Meghe Inst Med Sci Univ. 2018;13(1):63-65.

9. Lou SM, Rich AM, De Silva RK, et al. Pleomorphic adenoma of minor salivary gland. Oral Oncol Extra. 2006;42(4):170-172.

10. Farina A, Pelucchi S, Grandi E, et al. Histological subtypes of pleomorphic adenoma and age-frequency distribution. $\mathrm{Br} \mathrm{J}$ Oral Maxillofac Surg. 1999;37(2):154-155.

11. Verma P, Sachdeva SK, Verma KG, et al. Pleomorphic adenoma of cheek: A rare case report and review of literature. Indian J Dent Res. 2014;25(1):122-124.

12. Khandekar S, Dive A, Munde P, et al. Pleomorphic adenoma of the buccal salivary gland. J Oral Maxillofac Pathol. 2015;19(1):111.

13. Mannan R, Piplani S, Sharma S, et al. Cytological correlation of spectrum of head and neck lesions with epidemiological and diagnostic parameters. Indian J Pathol Oncol. 2017;4(1):92-97.

14. Sharma S, Mannan R, Bhasin T. Cytological diagnosis of deep-seated cellular hemangioma of the parotid gland by using cell button technique. J Cytol. 2016;33(3):174-176.

15. Rajpoot J, Sharma S, Arora R. Recurrent cutaneous leiomyosarcoma of the anterior abdominal wall: a rare tumor at an unusual site. Int $J$ Res Dermatol. 2017;3(1):151-154.

16. Sharma S, Sonali. Cutaneous metastasis from an asymptomatic breast cancer masquerading as an infected sebaceous cyst. Madridge J Dermtol Res. 2018;3(1):46-50.

17. Singh P, Mittal MK, Sharma S. Thyrolipoma: A Rare Thyroid Gland Entity. Nepalese Journal of Radiology. 2019;9(13):24-29.

18. Bhasin T, Mannan R, Manjari M, et al. Reproducibility of 'the Bethesda system for reporting thyroid cytopathology': A multicenter study with review of the literature. J Clin Diagn Res. 2013;7(6):1051-1054.
19. Sharma S, Mandal D, Yadav AK, et al. Primary thyroid lymphoma: a comprehensive summary of two cases. Int J Sci Rep. 2015;1(3):177-180.

20. Kaur J, Sharma S, Bhasin TS, et al. A rare incidental case of an occult breast carcinoma micrometastasis in papillary thyroid carcinoma: A view within a view. Thyroid Res Pract. 2018;15(3):142-146.

21. Sharma S, Singh M, Bhuyan G, et al. Extragonadal dysgerminoma presenting as neck metastasis and masquerading as a thyroid swelling. Clin Cancer Investig J. 2016;5(1):43-45.

22. Sharma S, Rana BP. Giant fibroadenoma of breast: a diagnostic dilemma in a middle aged woman. Adv Cytol Pathol. 2017;2(4):109-112.

23. Sharma S, Ahluwalia $\mathrm{C}$, Singh $\mathrm{M}$, et al. Diagnostic utility and efficacy of conventional versus surepath ${ }^{\circledR}$ liquid-based cytology in head and neck pathology: a study in an indian tertiary care hospital. Iran J Pathol. 2018;13(2):188-195.

24. Rajendran R. Tumors of the Salivary Glands. In: Rajendran R, Sivapathasundaram B, editors. Shafer's Text Book of Oral Pathology. 5th ed. New Delhi: Elsevier; 2006:311.

25. Speight PM. Update on diagnostic difficulties in lesions of the minor salivary glands. Head Neck Pathol. 2007;1(1):55-60.

26. Cohen MA. Pleomorphic adenoma of the cheek. Int J Oral Maxillofac Surg. 1986;15(6):777-779.

27. Geetha NT, Deepa BV, Umashankara KV, et al. Pleomorphic adenoma of minor salivary gland in the cheek. Int J Oral Health Sci. 2015;5(2):117120

28. Mubeen K, Vijayalakshmi KR, Pati AR, et al. Benign pleomorphic adenoma of minor salivary gland of palate. J Dent Oral Hyg. 2011;3:8288 .

29. Kini YK, Kalburge JV, Kharkar VR. A rare carcinoma ex pleomorphic adenoma of the buccal minor salivary gland causing a therapeutic dilemma. Indian J Cancer. 2016;53(1):18-19. 